\title{
A generalized Trudinger-Moser inequality on a compact Riemannian surface
}

\author{
Xiaobao Zhu \\ Department of Mathematics, Renmin University of China, Beijing 100872, P. R. China
}

\section{Abstract}

Let $(\Sigma, g)$ be a compact Riemannian surface. Let $\psi, h$ be two smooth functions on $\Sigma$ with $\int_{\Sigma} \psi d v_{g} \neq 0$ and $h \geq 0, h \neq \equiv$. In this paper, using a method of blowup analysis, we prove that the functional

$$
J^{\psi, h}(u)=\frac{1}{2} \int_{\Sigma}\left|\nabla_{g} u\right|^{2} d v_{g}+8 \pi \frac{1}{\int_{\Sigma} \psi d v_{g}} \int_{\Sigma} \psi u d v_{g}-8 \pi \log \int_{\Sigma} h e^{u} d v_{g}
$$

is bounded from below in $W^{1,2}(\Sigma, g)$. Moreover, we obtain a sufficient condition under which $J^{\psi, h}$ attains its infimum in $W^{1,2}(\Sigma, g)$. These results generalize the main results in [9] and [25].

Keywords: Trudinger-Moser inequality, variational method, blowup analysis, Kazdan-Warner equation

2010 MSC: $58 \mathrm{~J} 05$

\section{Introduction and main results}

Let $(\Sigma, g)$ be a compact Riemannian surface. Let $\psi, h$ be two smooth functions on $\Sigma$. In the celebrated paper [9], Ding-Jost-Li-Wang studied the functional $J^{\psi, h}$ in $W^{1,2}(\Sigma, g)$ when $\psi \equiv 1$ and $h>0$. Using a method of blowup analysis, they obtained a sufficient condition (8) with $\psi \equiv 1)$ under which $J^{1, h}$ attains its infimum in $W^{1,2}(\Sigma, g)$.

In this paper, we shall generalize Ding-Jost-Li-Wang's work [9]. Precisely, we prove the following:

Theorem 1. Let $(\Sigma, g)$ be a compact Riemannian surface. Let $\psi$ be a smooth function on $\Sigma$ satisfying $\int_{\Sigma} \psi d v_{g} \neq 0$. For any $u \in W^{1,2}(\Sigma, g)$ we have

$$
\int_{\Sigma} e^{u} d v_{g} \leq C_{\Sigma} \exp \left\{\frac{1}{16 \pi}\left\|\nabla_{g} u\right\|_{2}^{2}+\widetilde{u}\right\}
$$

where $\widetilde{u}=\frac{1}{\int_{\Sigma} \psi d v_{g}} \int_{\Sigma} \psi u d v_{g}$ and $C_{\Sigma}$ is a positive constant depending only on $(\Sigma, g)$.

Email address: zhuxiaobao@ruc.edu.cn (Xiaobao Zhu) 
Let $G_{y}(x)$ be the Green function which satisfies

$$
\left\{\begin{array}{l}
\Delta_{g} G_{y}(x)=8 \pi\left(\frac{\psi(x)}{\int_{\Sigma} \psi d v_{g}}-\delta_{y}(x)\right), x \in \Sigma \\
\int_{\Sigma} \psi G_{y} d v_{g}=0
\end{array}\right.
$$

In a normal coordinate system around $y, G_{y}(x)$ has the expression

$$
\begin{aligned}
G_{y}(x)= & -4 \log r+A_{y}+b_{1} r \cos \theta+b_{2} r \sin \theta \\
& +c_{1} r^{2} \cos ^{2} \theta+2 c_{2} r^{2} \cos \theta \sin \theta+c_{3} r^{2} \sin ^{2} \theta+O\left(r^{3}\right),
\end{aligned}
$$

where $r(x)=\operatorname{dist}(x, y)$ is the distance function from $x$ to $y$ on $(\Sigma, g)$.

To prove Theorem 1 we consider the perturbed functional

$$
J_{\epsilon}^{\psi, h}(u)=\frac{1}{2} \int_{\Sigma}\left|\nabla_{g} u\right|^{2} d v_{g}+8 \pi(1-\epsilon) \frac{1}{\int_{\Sigma} \psi d v_{g}} \int_{\Sigma} \psi u d v_{g}-8 \pi(1-\epsilon) \log \int_{\Sigma} h e^{u} d v_{g}
$$

for $\epsilon \in(0,1)$. In view of the classical Trudinger-Moser inequality (c.f. Lemma 4 below), $\forall \epsilon \in$ $(0,1), \exists u_{\epsilon} \in W^{1,2}(\Sigma, g)$, such that $J_{\epsilon}^{\psi, h}\left(u_{\epsilon}\right)=\inf _{u \in W^{1,2}(\Sigma, g)} J_{\epsilon}^{\psi, h}(u)$ and $u_{\epsilon}$ satisfies the EulerLagrange equation

$$
\Delta_{g} u_{\epsilon}=8 \pi(1-\epsilon)\left(\frac{\psi}{\int_{\Sigma} \psi d v_{g}}-\frac{h e^{u_{\epsilon}}}{\int_{\Sigma} h e^{u_{\epsilon}} d v_{g}}\right) .
$$

Without distinguishing sequence and its subsequences, there are two possibilities:

(i). If $u_{\epsilon}$ has a uniform bound in $W^{1,2}(\Sigma, g)$ (i.e., a bound does not depend on $\epsilon$ ), then $u_{\epsilon}$ converges to some $u_{0}$ in $W^{1,2}(\Sigma, g)$ and $u_{0}$ attains the infimum of $J^{\psi, h}$ in $W^{1,2}(\Sigma, g)$.

(ii). If $\left\|u_{\epsilon}\right\|_{W^{1,2}(\Sigma, g)} \rightarrow \infty$ as $\epsilon \rightarrow 0$, one calls $u_{\epsilon}$ blows up, we shall prove that

$$
\inf _{u \in W^{1,2}(\Sigma, g)} J^{\psi, h}(u) \geq-8 \pi-8 \pi \log \pi-4 \pi \max _{y \in \Sigma \backslash Z}\left(2 \log h(y)+A_{y}\right),
$$

where $Z=\{y \in \Sigma: h(y)=0\}$ and $A_{y}$ is a smooth function on $\Sigma$ defined in (3). Combining the results in $(i)$ and $(i i)$ one proves Theorem 1.

When $u_{\epsilon}$ blows up, we construct a blowup sequence $\left\{\phi_{\epsilon}\right\}_{\epsilon>0}$ (c.f. (46). By a direct calculation one obtains [56, letting $\epsilon \rightarrow 0$ we have

$$
\inf _{u \in W^{1,2}(\Sigma, g)} J^{\psi, h}(u) \leq \lim _{\epsilon \rightarrow 0} J^{\psi, h}\left(\phi_{\epsilon}\right)=-8 \pi-8 \pi \log \pi-4 \pi \max _{y \in \Sigma \backslash Z}\left(2 \log h(y)+A_{y}\right) .
$$

Combining (6) and (7) we have

Theorem 2. Let $(\Sigma, g)$ be a compact Riemannian surface. Let $J^{\psi, h}, J_{\epsilon}^{\psi, h}$ and $u_{\epsilon}$ be defined in (1), (4) and (5) respectively. If $u_{\epsilon}$ blows up, then we have

$$
\inf _{u \in W^{1,2}(\Sigma, g)} J^{\psi, h}(u)=-8 \pi-8 \pi \log \pi-4 \pi \max _{y \in \Sigma \backslash Z}\left(2 \log h(y)+A_{y}\right),
$$

where $A_{y}$ is defined in (3). 
In view of (56), if one has (8) below, then we have $J^{\psi, h}\left(\phi_{\epsilon}\right)<\inf _{u \in W^{1,2}(\Sigma, g)} J^{\psi, h}(u)$ for sufficiently small $\epsilon>0$. Then Theorem 2 tells us that no blowup happens, so $J^{\psi, h}$ achieves its infimum at some function $u \in W^{1,2}(\Sigma, g)$. Precisely, we obtain the following existence theorem.

Theorem 3. Let $(\Sigma, g)$ be a compact Riemannian surface, $K_{g}$ be its Gaussian curvature. Let $\psi, h$ be two smooth functions on $\Sigma$ with $\int_{\Sigma} \psi d v_{g} \neq 0$ and $h \geq 0, h \neq 0$. Denote $Z=\{y \in \Sigma: h(y)=0\}$. Suppose $2 \log h(y)+A_{y}$ attains its supremum in $\Sigma \backslash Z$ at $p$. Let $b_{1}(p)$ and $b_{2}(p)$ be the constants in the expression (3). In a normal coordinate system around $p$ we write $\nabla_{g} h(p)=\left(k_{1}(p), k_{2}(p)\right)$. If

$$
\begin{aligned}
& \Delta_{g} h(p)+2\left[b_{1}(p) k_{1}(p)+b_{2}(p) k_{2}(p)\right] \\
> & -\left[4 \pi\left(\frac{\psi(p)}{\int_{\Sigma} \psi d v_{g}}+1\right)+\left(b_{1}^{2}(p)+b_{2}^{2}(p)\right)-2 K_{g}(p)\right] h(p),
\end{aligned}
$$

then the infimum of the functional $J^{\psi, h}$ in $W^{1,2}(\Sigma, g)$ can be attained at some $u \in C^{\infty}(\Sigma)$ which satisfies

$$
\Delta_{g} u=8 \pi\left(\frac{\psi}{\int_{\Sigma} \psi d v_{g}}-\frac{h e^{u}}{\int_{\Sigma} h e^{u} d v_{g}}\right) .
$$

There are three motivations for the study of this paper:

Motivation 1. Ding-Jost-Li-Wang [9] studied existence of the Kazdan-Warner equation $\Delta_{g} u=$ $8 \pi-8 \pi h e^{u}$ on a compact Riemannian surface with volume 1 . First, they used a variational method to derive a lower bound for $J^{1, h}$ in $W^{1,2}(\Sigma, g)$; Then, they construct a blowup sequence $\left\{\phi_{\epsilon}\right\}_{\epsilon>0}$ to display that no blowup happens and obtained a sufficient condition ( $(8)$ with $\left.\psi \equiv 1\right)$ for the existence of the Kazdan-Warner equation (c.f. [14]). Our first motivation is to generalize these results, see Theorems 1 and 3

Motivation 2. Let $(\Sigma, g)$ be a compact Riemannian surface, $K_{g}$ be its Gaussian curvature. The Liouville energy of metric $\widetilde{g}=e^{u} g$ with respect to metric $g$ is represented as $L_{g}(\widetilde{g})=\int_{\Sigma}\left|\nabla_{g} u\right|^{2} d v_{g}+$ $4 \int_{\Sigma} K_{g} u d v_{g}$. When $(\Sigma, g)$ is a topological two sphere with volume $4 \pi$ and bounded curvature $K_{g}$, Chen-Zhu [8] proved that $L_{g}(\widetilde{g})$ is bounded from below in $W^{1,2}(\Sigma, g)$. Their proof is analytic, does not rely on the uniformization theorem and the Onofri inequality. In fact, this problem is equivalent to prove that $J^{1,1}$ is bounded from below in $W^{1,2}(\Sigma, g)$. Our second motivation is generalize Chen-Zhu's result to general Riemannian surfaces. This is our major motivation.

Motivation 3. Yang and the author [25] weakened the condition $h>0$ in [9] to $h \geq 0, h \neq \equiv 0$. Our third motivation is study existence of the generalized Kazdan-Warner equation (9) under this condition.

We refer the readers to $[20,23,15,19,3]$ and references therein for more relevant works.

Concluding remark: In this paper, we shall follow closely the lines of [9] and [25]. We would like to point out two things: First, in the proof of Theorem 1 when we estimate the integral $\int_{\Sigma}\left|\nabla_{g} u_{\epsilon}\right|^{2} d v_{g}$, we divide it into two parts

$$
\int_{B_{R r_{\epsilon}}\left(x_{\epsilon}\right)}\left|\nabla_{g} u\right|^{2} d v_{g} \quad \& \quad \int_{\Sigma \mid B_{R r_{\epsilon}}\left(x_{\epsilon}\right)}\left|\nabla_{g} u\right|^{2} d v_{g}
$$


instead of three parts

$$
\int_{B_{R_{\epsilon}}\left(x_{\epsilon}\right)}\left|\nabla_{g} u\right|^{2} d v_{g} \quad \& \quad \int_{B_{\delta}\left(x_{\epsilon}\right)}\left|\nabla_{g} u\right|^{2} d v_{g} \quad \& \quad \int_{\Sigma \backslash B_{\delta}\left(x_{\epsilon}\right)}\left|\nabla_{g} u\right|^{2} d v_{g}
$$

in [9], which can simplify the proof of Theorem 1] Second, when $u_{\epsilon}$ blows up, Ding-Jost-LiWang [9] proved that

$$
\inf _{u \in W^{1,2}(\Sigma, g)} J^{1, h}(u) \geq-8 \pi-8 \pi \log \pi-4 \pi \max _{y \in \Sigma}\left(2 \log h(y)+A_{y}\right) .
$$

we say more about this point. In fact, we shall prove in Theorem 2 that

$$
\inf _{u \in W^{1,2}(\Sigma, g)} J^{\psi, h}(u)=-8 \pi-8 \pi \log \pi-4 \pi \max _{y \in \Sigma \backslash Z}\left(2 \log h(y)+A_{y}\right)
$$

for a general smooth $\psi$ satisfies $\int_{\Sigma} \psi d v_{g} \neq 0$, where $Z=\{y \in \Sigma: h(y)=0\}$.

\section{Some main notations:}

- $\bar{u}=\frac{1}{\operatorname{Vol}_{g}(\Sigma)} \int_{\Sigma} u d v_{g} \quad \bullet \widetilde{u}=\frac{1}{\int_{\Sigma} \psi d v_{g}} \int_{\Sigma} \psi u d v_{g}$

- $\widetilde{X}=\left\{u \in W^{1,2}(\Sigma): \widetilde{u}=0\right\} \quad \bullet Z=\{y \in \Sigma: h(y)=0\}$

- $\|\cdot\|_{p}=\left(\int_{\Sigma}|\cdot|^{p} d v_{g}\right)^{1 / p}, L^{p}-\operatorname{norm}$ on $(\Sigma, g)$

- $J^{\psi, h}(u)=\frac{1}{2} \int_{\Sigma}\left|\nabla_{g} u\right|^{2} d v_{g}+8 \pi \frac{1}{\int_{\Sigma} \psi d v_{g}} \int_{\Sigma} \psi u d v_{g}-8 \pi \log \int_{\Sigma} h e^{u} d v_{g}$

- $J_{\epsilon}^{\psi, h}(u)=\frac{1}{2} \int_{\Sigma}\left|\nabla_{g} u\right|^{2} d v_{g}+8 \pi(1-\epsilon) \frac{1}{\int_{\Sigma} \psi d v_{g}} \int_{\Sigma} \psi u d v_{g}-8 \pi(1-\epsilon) \log \int_{\Sigma} h e^{u} d v_{g}$

The paper is organized as follows: In Section 2, we give three key inequalities. The proof of Theorem 11 is given in Section 3. In Sections 4 and 5, we divide the proof of Theorem 3 into two parts: $h>0$ and $h \geq 0, h \equiv 0$.

Throughout this paper, we use $C$ to denote a positive constant and its changes from line to line. We do not distinguish sequence and its subsequences in this paper.

\section{Three key inequalities}

In this section, we present three key inequalities which are very important in the following study.

2.1. The Trudinger-Moser inequality on a compact Riemannian surface

Lemma 4. ([11, 9]) Let $(\Sigma, g)$ be a compact Riemannian surface. For any $u \in W^{1,2}(\Sigma, g)$ we have

$$
\int_{\Sigma} e^{u} d v_{g} \leq C_{\Sigma} \exp \left\{\frac{1}{16 \pi}\left\|\nabla_{g} u\right\|_{2}^{2}+\bar{u}\right\},
$$

where $\bar{u}=\frac{1}{\operatorname{Vol}_{g}(\Sigma)} \int_{\Sigma} u d v_{g}$ and $C_{\Sigma}$ is a positive constant depending only on $(\Sigma, g)$. 
For improvements of the above Trudinger-Moser inequality, we refer the readers to AdimurthiDruet [1], Yang [21, 22], Lu-Yang [16], Wang-Ye [18], Yang-Zhu [24], Tintarev [17] and the author [26].

\subsection{The Poincaré type inequality}

Lemma 5. Let $(\Sigma, g)$ be a compact Riemannian surface. Let $\psi$ be a smooth function on $\Sigma$ satisfying $\int_{\Sigma} \psi d v_{g} \neq 0$. Assume $q>1$, then for any $u \in W^{1, q}(\Sigma, g)$ we have

$$
\left(\int_{\Sigma}|u-\widetilde{u}|^{q} d v_{g}\right)^{1 / q} \leq C_{\Sigma}\left(\int_{\Sigma}\left|\nabla_{g} u\right|^{q} d v_{g}\right)^{1 / q}
$$

where $\widetilde{u}=\frac{1}{\int_{\Sigma} \psi d v_{g}} \int_{\Sigma} \psi u d v_{g}$ and $C_{\Sigma}$ is a positive constant depending only on $(\Sigma, g)$.

Since the proof of Lemma 5 is completely analogous to the case that $\psi$ is a constant, we omit it here and refer the readers to Theorem 2.10 in [13].

\subsection{The Sobolev-Poincaré type inequality}

Lemma 6. Let $(\Sigma, g)$ be a compact Riemannian surface. Let $\psi$ be a smooth function on $\Sigma$ with $\int_{\Sigma} \psi d v_{g} \neq 0$. Assume $p \geq 1$, then for any $u \in W^{1,2}(\Sigma, g)$ we have

$$
\left(\int_{\Sigma}|u-\widetilde{u}|^{p} d v_{g}\right)^{1 / p} \leq C_{\Sigma}\left(\int_{\Sigma}\left|\nabla_{g} u\right|^{2} d v_{g}\right)^{1 / 2}
$$

where $\widetilde{u}=\frac{1}{\int_{\Sigma} \psi d v_{g}} \int_{\Sigma} \psi u d v_{g}$ and $C_{\Sigma}$ is a positive constant depending only on $(\Sigma, g)$.

Proof. The proof is standard. Suppose not, there exists a sequence of functions $\left\{u_{n}\right\}_{n=1}^{\infty} \subset$ $W^{1,2}(\Sigma, g)$ such that

$$
\left(\int_{\Sigma}\left|u_{n}-\widetilde{u}_{n}\right|^{p} d v_{g}\right)^{1 / p} \geq n\left(\int_{\Sigma}\left|\nabla_{g} u_{n}\right|^{2} d v_{g}\right)^{1 / 2}
$$

Let

$$
v_{n}=\frac{u_{n}-\widetilde{u}_{n}}{\left(\int_{\Sigma}\left|u_{n}-\widetilde{u}_{n}\right|^{p} d v_{g}\right)^{1 / p}} .
$$

Easily check can find that

$$
\left\|v_{n}\right\|_{p}=1,\left\|\nabla_{g} v_{n}\right\|_{2} \leq \frac{1}{n}, \widetilde{v}_{n}=0
$$

By Lemma 5 we have

$$
\left\|v_{n}\right\|_{2} \leq C
$$

So

$$
\left\|v_{n}\right\|_{W^{1,2(\Sigma, g)}} \leq C .
$$

Therefore,

$$
\begin{aligned}
& v_{n} \rightarrow v_{0} \text { weakly in } W^{1,2}(\Sigma, g), \\
& v_{n} \rightarrow v_{0} \text { strongly in } L^{q}(\Sigma, g)(\forall q \geq 1) .
\end{aligned}
$$


By the lower semi-continuous property of $\left\|\nabla_{g} \cdot\right\|_{2}^{2}$ one knows $\left\|\nabla_{g} v_{0}\right\|_{2}^{2}=0$, so $v_{0}$ is a constant. From (12), we have

$$
\widetilde{v}_{0}=0
$$

and

$$
\left\|v_{0}\right\|_{p}=1 .
$$

Since $v_{0}$ is a constant, (13) tells us that $v_{0} \equiv 0$. This is a contraction with (14). This ends the proof of the lemma.

\section{Proof of Theorem 1}

In this section, we shall derive the lower bound of $J^{\psi, h}$. As a consequence, we shall prove Theorem 1

Since $J_{\epsilon}^{\psi, h}(u+c)=J_{\epsilon}^{\psi, h}(u)$ and $J^{\psi, h}(u+c)=J^{\psi, h}(u)$ for any $\epsilon \in(0,1), c \in \mathbb{R}$ and any $u \in W^{1,2}(\Sigma, g)$, we have

$$
\inf _{u \in \widetilde{X}} J_{\epsilon}^{\psi, h}(u)=\inf _{u \in W^{1,2}(\Sigma, g)} J_{\epsilon}^{\psi, h}(u), \inf _{u \in \widetilde{X}} J^{\psi, h}(u)=\inf _{u \in W^{1,2}(\Sigma, g)} J^{\psi, h}(u),
$$

where $\widetilde{X}=\left\{u \in W^{1,2}(\Sigma): \widetilde{u}=0\right\}$. Therefore, we can without loss of generality assume that $u_{\epsilon} \in \widetilde{X}$. There are two possibilities:

Case a). $\left\|\nabla_{g} u_{\epsilon}\right\|_{2} \leq C$.

Since $u_{\epsilon} \in \widetilde{X}$, by the Poincaré inequality (11) we know $u_{\epsilon}$ is bounded in $W^{1,2}(\Sigma, g)$. Then we may assume

$$
\begin{aligned}
& u_{\epsilon} \rightarrow u_{0} \text { weakly in } W^{1,2}(\Sigma, g), \\
& u_{\epsilon} \rightarrow u_{0} \text { strongly in } L^{p}(\Sigma, g), \forall p \geq 1 .
\end{aligned}
$$

This together with the Trudinger-Moser inequality (10) and the Hölder inequality leads to

$$
\begin{aligned}
\int_{\Sigma} h\left(e^{u_{\epsilon}}-e^{u_{0}}\right) d v_{g} & =\int_{\Sigma} h \int_{0}^{1} \frac{d}{d t} e^{u_{0}+t\left(u_{\epsilon}-u_{0}\right)} d t d v_{g} \\
& =\int_{0}^{1} \int_{\Sigma} h e^{u_{0}+t\left(u_{\epsilon}-u_{0}\right)}\left(u_{\epsilon}-u_{0}\right) d v_{g} d t \\
& \rightarrow 0 \text { as } \epsilon \rightarrow 0 .
\end{aligned}
$$

From (15) and the Hölder inequality we have

$$
\int_{\Sigma} \psi\left(u_{\epsilon}-u_{0}\right) d v_{g} \rightarrow 0 \text { as } \epsilon \rightarrow 0
$$


The lower semi-continuous of $\left\|\nabla_{g} \cdot\right\|_{2}^{2}$ together with (15)-(17) gives us

$$
\inf _{u \in \widetilde{X}} J^{\psi, h}(u) \geq \liminf _{\epsilon \rightarrow 0} \inf _{u \in \widetilde{X}} J_{\epsilon}^{\psi, h}(u)=\liminf _{\epsilon \rightarrow 0} J_{\epsilon}^{\psi, h}\left(u_{\epsilon}\right) \geq J^{\psi, h}\left(u_{0}\right) .
$$

That is to say, $u_{0} \in \widetilde{X}$ attains the infimum of $J^{\psi, h}$ in $\widetilde{X}$ and satisfies (9). The elliptic regularity theory implies that $u_{0} \in C^{\infty}(\Sigma)$. The proof of Theorems 1 and 3 terminates in this case.

Case b). $\left\|\nabla_{g} u_{\epsilon}\right\|_{2} \rightarrow+\infty$ as $\epsilon \rightarrow 0$.

Though the $L^{2}$-norm of the gradient of $u_{\epsilon}$ is infinity, we have

Lemma 7. For any $1<q<2,\left\|\nabla_{g} u_{\epsilon}\right\|_{q} \leq C$.

Proof. Let $q^{\prime}=q /(q-1)>2$, by equation (5) we have

$$
\begin{aligned}
\left\|\nabla_{g} u_{\epsilon}\right\|_{q} & \leq \sup _{\|\zeta\|_{W^{1, q^{\prime}(\Sigma)} \leq 1,} \int_{\Sigma} \zeta d v_{g}=0} \int_{\Sigma} \nabla_{g} u_{\epsilon} \nabla_{g} \zeta d v_{g} \\
& =\sup _{\|\zeta\|_{W^{1, q^{\prime}(\Sigma)}} \leq 1, \int_{\Sigma} \zeta d v_{g}=0} \int_{\Sigma} 8 \pi(1-\epsilon)\left(-\frac{\psi}{\int_{\Sigma} \psi d v_{g}}+\frac{h e^{u_{\epsilon}}}{\int_{\Sigma} h e^{u_{\epsilon}} d v_{g}}\right) \zeta d v_{g} \\
& \leq C,
\end{aligned}
$$

where in the last inequality we have used the Sobolev embedding $W^{1, q^{\prime}}(\Sigma, g) \hookrightarrow C^{0}(\Sigma)$.

Denote $\lambda_{\epsilon}=\int_{\Sigma} h e^{u_{\epsilon}} d v_{g}$. We have

Lemma 8. $\lim \inf _{\epsilon \rightarrow 0} \lambda_{\epsilon}>0$.

Proof.

$$
J_{\epsilon}^{\psi, h}\left(u_{\epsilon}\right)=\inf _{u \in \widetilde{X}} J_{\epsilon}^{\psi, h}(u) \leq J_{\epsilon}^{\psi, h}(0) \leq-8 \pi(1-\epsilon) \log \int_{\Sigma} h d v_{g} .
$$

If $\liminf _{\epsilon \rightarrow 0} \lambda_{\epsilon}=0$, then up to a subsequence we have

$$
J_{\epsilon}^{\psi, h}\left(u_{\epsilon}\right)=\frac{1}{2} \int_{\Sigma}\left|\nabla_{g} u_{\epsilon}\right|^{2} d v_{g}-8 \pi(1-\epsilon) \log \lambda_{\epsilon} \rightarrow+\infty
$$

as $\epsilon \rightarrow 0$, which contradicts (18). This ends the proof of Lemma 8 ,

$$
\text { Let } c_{\epsilon}=\max _{\Sigma} u_{\epsilon}=u_{\epsilon}\left(x_{\epsilon}\right) \text {. Suppose } x_{\epsilon} \rightarrow p \text { as } \epsilon \rightarrow 0 \text {, then }
$$

Lemma 9. $c_{\epsilon} \rightarrow+\infty$ as $\epsilon \rightarrow 0$. Furthermore, we have $\lambda_{\epsilon}^{-1} e^{c_{\epsilon}} \rightarrow+\infty$ as $\epsilon \rightarrow 0$.

Proof. Multiplying both sides of the equation (5) by $u_{\epsilon}$ and integrating both sides on $(\Sigma, g)$, we have

$$
\int_{\Sigma}\left|\nabla_{g} u_{\epsilon}\right|^{2} d v_{g} \leq 8 \pi(1-\epsilon) c_{\epsilon}
$$

This implies $c_{\epsilon} \rightarrow+\infty$ as $\epsilon \rightarrow 0$ since $\left\|\nabla_{g} u_{\epsilon}\right\|_{2} \rightarrow+\infty$ as $\epsilon \rightarrow 0$. 
By Lemmas 4 and 5 one has

$$
\begin{aligned}
\lambda_{\epsilon}=\int_{\Sigma} h e^{u_{\epsilon}} d v_{g} & \leq C \exp \left\{\frac{1}{16 \pi}\left\|\nabla_{g} u_{\epsilon}\right\|_{2}^{2}+\bar{u}_{\epsilon}\right\} \\
& \leq C \exp \left\{\left(\frac{1}{16 \pi}+\delta\right)\left\|\nabla_{g} u_{\epsilon}\right\|_{2}^{2}+C_{\delta}\right\} .
\end{aligned}
$$

Substituting (19) into (20) and choosing $\delta=\frac{1+2 \epsilon}{32 \pi(1-\epsilon)}$, then

$$
\lambda_{\epsilon}^{-1} e^{c_{\epsilon}} \geq C e^{\frac{1}{4} c_{\epsilon}} \rightarrow+\infty \text { as } \epsilon \rightarrow 0 .
$$

This ends the proof of Lemma 9

Choosing a local coordinate system $(U, z)$ around $p$, which satisfies $z(p)=0$. Let $r_{\epsilon}=$ $\sqrt{\lambda_{\epsilon}} e^{-c_{\epsilon} / 2}$ and define

$$
\varphi_{\epsilon}(x)=u_{\epsilon}\left(z\left(x_{\epsilon}\right)+r_{\epsilon} x\right)-\lambda_{\epsilon}, \quad x \in \mathbb{B}_{R}(0) \subset z(U) .
$$

Then in $\mathbb{B}_{R}(0)$,

$$
\begin{aligned}
\Delta_{g} \varphi_{\epsilon}(x) & =8 \pi(1-\epsilon)\left(\frac{\psi\left(z\left(x_{\epsilon}\right)+r_{\epsilon} x\right)}{\int_{\Sigma} \psi d v_{g}} r_{\epsilon}^{2}-h\left(z\left(x_{\epsilon}\right)+r_{\epsilon} x\right) e^{\varphi_{\epsilon}(x)}\right) \\
& :=f_{\epsilon}(x) .
\end{aligned}
$$

We have the following asymptotic phenomenon of $u_{\epsilon}$ near the blowup point $p$.

\section{Lemma 10.}

$$
\varphi_{\epsilon}(x) \rightarrow \varphi_{0}(x)=-2 \log \left(1+\pi h(p)|x|^{2}\right)
$$

in $C_{\text {loc }}^{1}\left(\mathbb{R}^{2}\right)$ as $\epsilon \rightarrow 0$.

Proof. Since $f_{\epsilon} \in L^{\infty}\left(\mathbb{B}_{R}(0)\right)$, by Theorem 9.15 in [12] we can consider the equation

$$
\left\{\begin{array}{l}
\Delta_{g} \varphi_{\epsilon}^{1}(x)=f_{\epsilon}(x), \quad x \in \mathbb{B}_{R}(0), \\
\left.\varphi_{\epsilon}^{1}\right|_{\partial \mathbb{B}_{R}(0)}=0 .
\end{array}\right.
$$

Let $\varphi_{\epsilon}^{2}=\varphi_{\epsilon}-\varphi_{\epsilon}^{1}$, then $\Delta_{g} \varphi_{\epsilon}^{2}=0$. Since $\varphi_{\epsilon} \leq 0, f_{\epsilon}$ is bounded in $\mathbb{B}_{R}(0)$. The elliptic estimates together with $W_{0}^{2, p}\left(\mathbb{B}_{R}(0)\right) \hookrightarrow C\left(\overline{\mathbb{B}_{R}(0)}\right)$ give $\sup _{\mathbb{B}_{R}(0)}\left|\varphi_{\epsilon}^{1}\right| \leq C$. Because $\varphi_{\epsilon} \leqslant 0$, we have $\sup _{\mathbb{B}_{R}(0)} \varphi_{\epsilon}^{2} \leqslant C$. The Harnack inequality yields that $\sup _{\mathbb{B}_{\frac{R}{2}}(0)}\left|\varphi_{\epsilon}^{2}\right| \leqslant C$, because $\varphi_{\epsilon}^{2}(0)$ is bounded. Therefore $\sup _{\mathbb{B}_{\frac{R}{2}}(0)}\left|\varphi_{\epsilon}\right| \leqslant C$.

By the elliptic estimates, we can show that $\varphi_{\epsilon}(x) \rightarrow \varphi_{0}(x)$ in $C_{l o c}^{1}\left(\mathbb{R}^{2}\right)$ as $\epsilon \rightarrow 0$, where $\varphi_{0}(x)$ satisfies

$$
\left\{\begin{array}{l}
\Delta_{\mathbb{R}^{2}} \varphi_{0}=-8 \pi h(p) e^{\varphi_{0}} \\
\varphi_{0}(0)=0 \\
\int_{\mathbb{R}^{2}} h(p) e^{\varphi_{0}} d x \leq 1
\end{array}\right.
$$

By Chen-Li's classification theorem [6] we know

$$
\varphi_{0}(x)=-2 \log \left(1+\pi h(p)|x|^{2}\right) .
$$

This is the end of the proof of Lemma 10. 
Away from the blowup point $p$, we have

Lemma 11. For any $\Omega \subset \subset \Sigma \backslash\{p\}$, we have $\left\|u_{\epsilon}\right\|_{L^{\infty}(\Omega)} \leq C$.

Proof. Let $\Omega \subset \subset \Sigma \backslash\{p\}$. We choose another two compact sets $\Omega_{1}$ and $\Omega_{2}$ in $\Sigma \backslash\{p\}$ such that $\Omega \subset \subset \Omega_{1} \subset \subset \Omega_{2} \subset \subset \Sigma \backslash\{p\}$. Calculating directly, one knows in Lemma 10

$$
\lim _{R \rightarrow+\infty} \lim _{\epsilon \rightarrow 0} \lambda_{\epsilon}^{-1} \int_{B_{R r_{\epsilon}}\left(x_{\epsilon}\right)} h e^{u_{\epsilon}} d v_{g}=\int_{\mathbb{R}^{2}} h(p) e^{\varphi_{0}} d x=1
$$

Then by (22) one has

$$
\lim _{R \rightarrow+\infty} \lim _{\epsilon \rightarrow 0} \lambda_{\epsilon}^{-1} \int_{\Sigma \backslash B_{R r_{\epsilon}}\left(x_{\epsilon}\right)} h e^{u_{\epsilon}} d v_{g}=0
$$

So we have

$$
\lim _{\epsilon \rightarrow 0} \lambda_{\epsilon}^{-1} \int_{\Omega_{2}} h e^{u_{\epsilon}} d v_{g}=0 .
$$

Assume $u_{\epsilon}^{1}$ be the unique solution of

$$
\left\{\begin{array}{l}
\Delta_{g} u_{\epsilon}^{1}=-8 \pi(1-\epsilon) \lambda_{\epsilon}^{-1} h e^{u_{\epsilon}} \text { in } \Omega_{2}, \\
u_{\epsilon}^{1}=0 \text { on } \partial \Omega_{2} .
\end{array}\right.
$$

From (24) and Theorem 1 in [4] one knows that for some $q \in(1,2)$, we have

$$
\int_{\Omega_{2}} e^{q\left|u_{\epsilon}^{1}\right|} d v_{g} \leq C
$$

It follows that in (25) one has

$$
\left\|u_{\epsilon}^{1}\right\|_{L^{q}\left(\Omega_{2}\right)} \leq C \text {. }
$$

Let $u_{\epsilon}^{2}=u_{\epsilon}-u_{\epsilon}^{1}$. Then $\Delta_{g} u_{\epsilon}^{2}=8 \pi(1-\epsilon) \psi / \int_{\Sigma} \psi d v_{g}$ in $\Omega_{2}$. It follows from the interior $L^{p}$-estimates (c.f. Theorem 8.17 in [12]) and Lemma 7 that

$$
\begin{aligned}
\left\|u_{\epsilon}^{2}\right\|_{L^{\infty}\left(\Omega_{1}\right)} & \leq C\left\|u_{\epsilon}^{2}\right\|_{L^{q}\left(\Omega_{2}\right)} \\
& \leq C\left(\left\|u_{\epsilon}\right\|_{L^{q}\left(\Omega_{2}\right)}+\left\|u_{\epsilon}^{1}\right\|_{L^{q}\left(\Omega_{2}\right)}\right) \\
& \leq C\left(\left\|\nabla_{g} u_{\epsilon}\right\|_{L^{q}(\Sigma)}+\left\|u_{\epsilon}^{1}\right\|_{L^{q}\left(\Omega_{2}\right)}\right) \\
& \leq C .
\end{aligned}
$$

By combining (26) and (27) we have

$$
\int_{\Omega_{1}} e^{p u_{\epsilon}} d v_{g}=\int_{\Omega_{1}} e^{p u_{\epsilon}^{1}} e^{p u_{\epsilon}^{2}} d v_{g} \leq C .
$$

Using the standard elliptic estimates to equation (25), one obtains

$$
\left\|u_{\epsilon}^{1}\right\|_{L^{\infty}(\Omega)} \leq C \text {. }
$$

Thus,

$$
\left\|u_{\epsilon}\right\|_{L^{\infty}(\Omega)} \leq C
$$

This ends the proof of Lemma 11 
Remark 12. In fact, one can show that $u_{\epsilon} \rightarrow G_{p}$ in $C_{l o c}^{1}(\Sigma \backslash\{p\})$ as $\epsilon \rightarrow 0$, like in [9]. However, we do not need this fact, so we omit it here.

To complete the proof of Theorem 1, we still need a lower bound for $u_{\epsilon}$ away from the maximum point $x_{\epsilon}$.

Similar to [9], we have the following lemma by the maximum principle.

Lemma 13. For any fixed $R>0$, let $r_{\epsilon}=\sqrt{\lambda_{\epsilon}} e^{-c_{\epsilon} / 2}$. Then for any $y \in \Sigma \backslash B_{R r_{\epsilon}}\left(x_{\epsilon}\right)$, we have

$$
u_{\epsilon}(y)-G_{x_{\epsilon}}(y) \geq-c_{\epsilon}+2 \log \lambda_{\epsilon}-2 \log \pi-2 \log h(p)-A_{p}+o_{\epsilon}(1)+o_{R}(1),
$$

where $o_{\epsilon}(1) \rightarrow 0$ as $\epsilon \rightarrow 0$ and $o_{R}(1) \rightarrow 0$ as $R \rightarrow+\infty$.

Proof. By (5) and (2), we have for any $y \in \Sigma \backslash B_{R r_{\epsilon}}\left(x_{\epsilon}\right)$

$$
\Delta_{g}\left(u_{\epsilon}-G_{x_{\epsilon}}\right)=-8 \pi \epsilon \frac{\psi}{\int_{\Sigma} \psi d v_{g}}-8 \pi(1-\epsilon) \lambda_{\epsilon}^{-1} h e^{u_{\epsilon}} .
$$

Notice that one needs to deal with the term $-8 \pi \epsilon \psi / \int_{\Sigma} \psi d v_{g}$ which sign is unknown. Employing the trick introduced by Chen-Zhu (c.f. Lemma 3.5 in [8]), we define

$$
\psi^{\prime}= \begin{cases}\frac{\psi}{\int_{\Sigma} \psi d v_{g}}, & \text { if } \frac{\psi}{\int_{\Sigma} \psi d v_{g}} \leq 0, \\ \phi \frac{\psi}{\int_{\Sigma} \psi d v_{g}}, & \text { if } \frac{\psi}{\int_{\Sigma} \psi d v_{g}}>0,\end{cases}
$$

where $\phi(x) \in[0,1]$ is a measurable function on $\Sigma$ such that $\int_{\Sigma} \psi^{\prime} d v_{g}=0$. Assume $\Delta_{g} \zeta=\psi^{\prime}$ on $\Sigma$. Then $\zeta$ is bounded on $\Sigma$. Consider the function $u_{\epsilon}-G_{x_{\epsilon}}+8 \pi \epsilon \zeta$, we have

$$
\Delta_{g}\left(u_{\epsilon}-G_{x_{\epsilon}}+8 \pi \epsilon \zeta\right)=-8 \pi \epsilon\left(\frac{\psi}{\int_{\Sigma} \psi d v_{g}}-\psi^{\prime}\right)-8 \pi(1-\epsilon) \lambda_{\epsilon}^{-1} h e^{u_{\epsilon}} \leq 0, \quad y \in \Sigma \backslash B_{R r_{\epsilon}}\left(x_{\epsilon}\right) .
$$

Lemma 10 together with (3) tells us that

$$
\left.\left(u_{\epsilon}-G_{x_{\epsilon}}+8 \pi \epsilon \zeta\right)\right|_{\partial B_{R_{\epsilon}}\left(x_{\epsilon}\right)}=-c_{\epsilon}+2 \log \lambda_{\epsilon}-2 \log \pi-2 \log h(p)-A_{x_{\epsilon}}+o_{\epsilon}(1)+o_{R}(1) .
$$

Then by the maximum principle we know

$$
u_{\epsilon}-G_{x_{\epsilon}}+8 \pi \epsilon \zeta \geq-c_{\epsilon}+2 \log \lambda_{\epsilon}-2 \log \pi-2 \log h(p)-A_{x_{\epsilon}}+o_{\epsilon}(1)+o_{R}(1) .
$$

Since $\zeta$ is bounded and $A_{x_{\epsilon}} \rightarrow A_{p}$ as $\epsilon \rightarrow 0$, we have

$$
u_{\epsilon}-G_{x_{\epsilon}} \geq-c_{\epsilon}+2 \log \lambda_{\epsilon}-2 \log \pi-2 \log h(p)-A_{p}+o_{\epsilon}(1)+o_{R}(1) .
$$

This ends the proof of the lemma.

We are now ready to estimate $J_{\epsilon}^{\psi, h}\left(u_{\epsilon}\right)$ from below and give the proof of Theorem 1

Proof of Theorem 1 Recall that $r_{\epsilon}=\sqrt{\lambda_{\epsilon}} e^{-c_{\epsilon} / 2}$, for any fixed $R>0$, we have

$$
\int_{\Sigma}\left|\nabla_{g} u_{\epsilon}\right|^{2} d v_{g}=\int_{\Sigma \backslash B_{R r_{\epsilon}}\left(x_{\epsilon}\right)}\left|\nabla_{g} u_{\epsilon}\right|^{2} d v_{g}+\int_{B_{R r_{\epsilon}}\left(x_{\epsilon}\right)}\left|\nabla_{g} u_{\epsilon}\right|^{2} d v_{g} .
$$


From Lemma 10 one knows

$$
\begin{aligned}
\int_{B_{R r_{\epsilon}}\left(x_{\epsilon}\right)}\left|\nabla_{g} u_{\epsilon}\right|^{2} d v_{g} & =\int_{B_{R}(0)}\left|\nabla_{\mathbb{R}^{2}} \varphi_{0}\right|^{2} d x+o_{\epsilon}(1) \\
& =16 \pi \log \left(1+\pi h(p) R^{2}\right)-16 \pi+o_{\epsilon}(1)+o_{R}(1) .
\end{aligned}
$$

By the equation of $u_{\epsilon}$ (c.f. (5) we have

$$
\begin{aligned}
\int_{\Sigma \backslash B_{R r_{\epsilon}}\left(x_{\epsilon}\right)}\left|\nabla_{g} u_{\epsilon}\right|^{2} d v_{g}= & -\int_{\Sigma \backslash B_{R r_{\epsilon}}\left(x_{\epsilon}\right)} u_{\epsilon} \Delta_{g} u_{\epsilon} d v_{g}-\int_{\partial B_{R r_{\epsilon}}\left(x_{\epsilon}\right)} u_{\epsilon} \frac{\partial u_{\epsilon}}{\partial n} d s_{g} \\
= & 8 \pi(1-\epsilon) \lambda_{\epsilon}^{-1} \int_{\Sigma \backslash B_{R r_{\epsilon}}\left(x_{\epsilon}\right)} u_{\epsilon} h e^{u_{\epsilon}} d v_{g} \\
& -\frac{8 \pi(1-\epsilon)}{\int_{\Sigma} \psi d v_{g}} \int_{\Sigma \backslash B_{R r_{\epsilon}}\left(x_{\epsilon}\right)} \psi u_{\epsilon} d v_{g}-\int_{\partial B_{R r_{\epsilon}}\left(x_{\epsilon}\right)} u_{\epsilon} \frac{\partial u_{\epsilon}}{\partial n} d s_{g} .
\end{aligned}
$$

Lemma 13 leads to

$$
\begin{aligned}
& \quad 8 \pi(1-\epsilon) \lambda_{\epsilon}^{-1} \int_{\Sigma \backslash B_{R r_{\epsilon}}\left(x_{\epsilon}\right)} u_{\epsilon} h e^{u_{\epsilon}} d v_{g} \\
& \geq 8 \pi(1-\epsilon) \lambda_{\epsilon}^{-1} \int_{\Sigma \backslash B_{R r_{\epsilon}}\left(x_{\epsilon}\right)} G_{x_{\epsilon}} h e^{u_{\epsilon}} d v_{g}-8 \pi(1-\epsilon) \lambda_{\epsilon}^{-1}\left(c_{\epsilon}-2 \log \lambda_{\epsilon}\right) \int_{\Sigma \backslash B_{R r_{\epsilon}}\left(x_{\epsilon}\right)} h e^{u_{\epsilon}} d v_{g} \\
& \quad+8 \pi(1-\epsilon) \lambda_{\epsilon}^{-1} \int_{\Sigma \backslash B_{R r_{\epsilon}}\left(x_{\epsilon}\right)}\left(-2 \log \pi-2 \log h(p)-A_{p}+o_{\epsilon}(1)+o_{R}(1)\right) h e^{u_{\epsilon}} d v_{g} .
\end{aligned}
$$

From (5) and (2) one has

$$
\begin{aligned}
& 8 \pi(1-\epsilon) \lambda_{\epsilon}^{-1} \int_{\Sigma \backslash B_{R r_{\epsilon}}\left(x_{\epsilon}\right)} G_{x_{\epsilon}} h e^{u_{\epsilon}} d v_{g} \\
= & \int_{\Sigma \backslash B_{R r_{\epsilon}}\left(x_{\epsilon}\right)} G_{x_{\epsilon}}\left(-\Delta_{g} u_{\epsilon}+8 \pi(1-\epsilon) \frac{\psi}{\int_{\Sigma} \psi d v_{g}}\right) d v_{g} \\
= & \frac{8 \pi}{\int_{\Sigma} \psi d v_{g}} \int_{B_{R r_{\epsilon}}\left(x_{\epsilon}\right)} u_{\epsilon} \psi d v_{g}-\frac{8 \pi(1-\epsilon)}{\int_{\Sigma} \psi d v_{g}} \int_{B_{R r_{\epsilon}}\left(x_{\epsilon}\right)} G_{x_{\epsilon}} \psi d v_{g} \\
& +\int_{\partial B_{R r_{\epsilon}}\left(x_{\epsilon}\right)} G_{x_{\epsilon}} \frac{\partial u_{\epsilon}}{\partial n} d s_{g}-\int_{\partial B_{R r_{\epsilon}}\left(x_{\epsilon}\right)} u_{\epsilon} \frac{\partial G_{x_{\epsilon}}}{\partial n} d s_{g} .
\end{aligned}
$$

It also follows from (5) that

$$
\begin{aligned}
& -8 \pi(1-\epsilon) \lambda_{\epsilon}^{-1}\left(c_{\epsilon}-2 \log \lambda_{\epsilon}\right) \int_{\Sigma \backslash B_{R r_{\epsilon}}\left(x_{\epsilon}\right)} h e^{u_{\epsilon}} d v_{g} \\
= & \left(c_{\epsilon}-2 \log \lambda_{\epsilon}\right) \int_{\partial B_{R r_{\epsilon}}\left(x_{\epsilon}\right)} \frac{\partial u_{\epsilon}}{\partial n} d s_{g}-\left(c_{\epsilon}-2 \log \lambda_{\epsilon}\right) \frac{8 \pi(1-\epsilon)}{\int_{\Sigma} \psi d v_{g}} \int_{\Sigma \backslash B_{R r_{\epsilon}}\left(x_{\epsilon}\right)} \psi d v_{g} .
\end{aligned}
$$

It follows from 23) that

$$
8 \pi(1-\epsilon) \lambda_{\epsilon}^{-1} \int_{\Sigma \backslash B_{R r_{\epsilon}}\left(x_{\epsilon}\right)}\left(-2 \log \pi-2 \log h(p)-A_{p}+o_{\epsilon}(1)+o_{R}(1)\right) h e^{u_{\epsilon}} d v_{g}=o_{\epsilon}(1) .
$$


Inserting (33)-(35) into (32) one obtains that

$$
\begin{aligned}
& \quad 8 \pi(1-\epsilon) \lambda_{\epsilon}^{-1} \int_{\Sigma \backslash B_{R r_{\epsilon}}\left(x_{\epsilon}\right)} u_{\epsilon} h e^{u_{\epsilon}} d v_{g} \\
& \geq \frac{8 \pi}{\int_{\Sigma} \psi d v_{g}} \int_{B_{R_{\epsilon} \epsilon}\left(x_{\epsilon}\right)} u_{\epsilon} \psi d v_{g}-\frac{8 \pi(1-\epsilon)}{\int_{\Sigma} \psi d v_{g}} \int_{B_{R r_{\epsilon}}\left(x_{\epsilon}\right)} G_{x_{\epsilon}} \psi d v_{g} \\
& \quad+\int_{\partial B_{R_{\epsilon}}\left(x_{\epsilon}\right)} G_{x_{\epsilon}} \frac{\partial u_{\epsilon}}{\partial n} d s_{g}-\int_{\partial B_{R_{\epsilon}}\left(x_{\epsilon}\right)} u_{\epsilon} \frac{\partial G_{x_{\epsilon}}}{\partial n} d s_{g} \\
& \quad+\left(c_{\epsilon}-2 \log \lambda_{\epsilon}\right) \int_{\partial B_{R r_{\epsilon}}\left(x_{\epsilon}\right)} \frac{\partial u_{\epsilon}}{\partial n} d s_{g} \\
& \quad-\left(c_{\epsilon}-2 \log \lambda_{\epsilon}\right) \frac{8 \pi(1-\epsilon)}{\int_{\Sigma} \psi d v_{g}} \int_{\Sigma \backslash B_{R r_{\epsilon}}\left(x_{\epsilon}\right)} \psi d v_{g}+o_{\epsilon}(1) .
\end{aligned}
$$

Substituting (36) into (31) we have

$$
\begin{aligned}
\int_{\Sigma \mid B_{R r_{\epsilon}}\left(x_{\epsilon}\right)}\left|\nabla_{g} u_{\epsilon}\right|^{2} d v_{g} \geq & \frac{8 \pi}{\int_{\Sigma} \psi d v_{g}} \int_{B_{R r_{\epsilon}}\left(x_{\epsilon}\right)} u_{\epsilon} \psi d v_{g}-\frac{8 \pi(1-\epsilon)}{\int_{\Sigma} \psi d v_{g}} \int_{B_{R r_{\epsilon}}\left(x_{\epsilon}\right)} G_{x_{\epsilon}} \psi d v_{g} \\
& +\int_{\partial B_{R_{\epsilon} \epsilon}\left(x_{\epsilon}\right)} G_{x_{\epsilon}} \frac{\partial u_{\epsilon}}{\partial n} d s_{g}-\int_{\partial B_{R r_{\epsilon}}\left(x_{\epsilon}\right)} u_{\epsilon} \frac{\partial G_{x_{\epsilon}}}{\partial n} d s_{g} \\
& +\left(c_{\epsilon}-2 \log \lambda_{\epsilon}\right) \int_{\partial B_{R_{\epsilon}}\left(x_{\epsilon}\right)} \frac{\partial u_{\epsilon}}{\partial n} d s_{g} \\
& -\left(c_{\epsilon}-2 \log \lambda_{\epsilon}\right) \frac{8 \pi(1-\epsilon)}{\int_{\Sigma} \psi d v_{g}} \int_{\Sigma \backslash B_{R r_{\epsilon}}\left(x_{\epsilon}\right)} \psi d v_{g} \\
& +\frac{8 \pi(1-\epsilon)}{\int_{\Sigma} \psi d v_{g}} \int_{B_{R r_{\epsilon}}\left(x_{\epsilon}\right)} \psi u_{\epsilon} d v_{g}-\int_{\partial B_{R r_{\epsilon}}\left(x_{\epsilon}\right)} u_{\epsilon} \frac{\partial u_{\epsilon}}{\partial n} d s_{g}+o_{\epsilon}(1) .
\end{aligned}
$$

Lemma 10 tells us that

$$
\int_{B_{R r_{\epsilon}}\left(x_{\epsilon}\right)} u_{\epsilon} \psi d v_{g}=o_{\epsilon}(1) .
$$

From (3) one knows

$$
\int_{B_{R r_{\epsilon}}\left(x_{\epsilon}\right)} G_{x_{\epsilon}} \psi d v_{g}=o_{\epsilon}(1) .
$$

Using Lemmas 10 and 13, one has

$$
\begin{aligned}
& -\int_{\partial B_{R r_{\epsilon}\left(x_{\epsilon}\right)}} \frac{\partial u_{\epsilon}}{\partial n}\left(u_{\epsilon}-G_{x_{\epsilon}}+c_{\epsilon}-2 \log \lambda_{\epsilon}\right) d s_{g} \\
\geq & \frac{8 \pi^{2} h(p) R^{2}}{1+\pi h(p) R^{2}}\left(-2 \log \pi-2 \log h(p)-A_{x_{\epsilon}}\right)+o_{\epsilon}(1)+o_{R}(1) \\
= & -16 \pi \log \pi-16 \pi \log h(p)-8 \pi A_{p}+o_{\epsilon}(1)+o_{R}(1) .
\end{aligned}
$$


In view of (21) and (3) we have

$$
\begin{aligned}
-\int_{\partial B_{R r_{\epsilon}}\left(x_{\epsilon}\right)} u_{\epsilon} \frac{\partial G_{x_{\epsilon}}}{\partial n} d s_{g} & =-\left(c_{\epsilon}-2 \log \left(1+\pi h(p) R^{2}\right)+o_{\epsilon}(1)\right)\left(-8 \pi+O\left(R r_{\epsilon}\right)\right) \\
& =8 \pi c_{\epsilon}-16 \pi \log \left(1+\pi h(p) R^{2}\right)+o_{\epsilon}(1)+o_{R}(1) .
\end{aligned}
$$

It is clear that by Lemmas 8 and 9

$$
-\left(c_{\epsilon}-2 \log \lambda_{\epsilon}\right) \frac{8 \pi(1-\epsilon)}{\int_{\Sigma} \psi d v_{g}} \int_{\Sigma \backslash B_{R r_{\epsilon}}\left(x_{\epsilon}\right)} \psi d v_{g}=-8 \pi(1-\epsilon)\left(c_{\epsilon}-2 \log \lambda_{\epsilon}\right)+o_{\epsilon}(1) .
$$

Therefore, by inserting (38)-(42) into (37) we obtain

$$
\begin{aligned}
\int_{\Sigma \backslash B_{R_{\epsilon}}\left(x_{\epsilon}\right)}\left|\nabla_{g} u_{\epsilon}\right|^{2} d v_{g} \geq & -16 \pi \log \pi-16 \pi \log h(p)-8 \pi A_{p} \\
& +8 \pi \epsilon c_{\epsilon}-16 \pi \log \left(1+\pi h(p) R^{2}\right) \\
& +16 \pi(1-\epsilon) \log \lambda_{\epsilon}+o_{\epsilon}(1)+o_{R}(1) .
\end{aligned}
$$

Substituting (30) and (43) into (29) one has

$$
\begin{aligned}
J_{\epsilon}^{\psi, h}\left(u_{\epsilon}\right)= & \frac{1}{2} \int_{\Sigma}\left|\nabla_{g} u_{\epsilon}\right|^{2} d v_{g}-8 \pi(1-\epsilon) \log \lambda_{\epsilon} \\
\geq & -8 \pi-8 \pi \log \pi-8 \pi \log h(p)-4 \pi A_{p} \\
& +4 \pi \epsilon c_{\epsilon}+o_{\epsilon}(1)+o_{R}(1)
\end{aligned}
$$

Letting $\epsilon \rightarrow 0$ first, and then $R \rightarrow+\infty$ in (44), we have

$$
\begin{aligned}
\inf _{u \in W^{1,2}(\Sigma, g)} J^{\psi, h}(u)=\lim _{\epsilon \rightarrow 0} J_{\epsilon}^{\psi, h}\left(u_{\epsilon}\right) & \geq-8 \pi-8 \pi \log \pi-8 \pi \log h(p)-4 \pi A_{p} \\
& \geq-8 \pi-8 \pi \log \pi-4 \pi \max _{y \in \Sigma \mid Z}\left(2 \log h(y)+A_{y}\right) .
\end{aligned}
$$

Then Theorem 1 follows directly.

\section{Proof of Theorem 3—Part I: $h>0$}

In this section, we shall construct a blowup sequence $\left\{\phi_{\epsilon}\right\}_{\epsilon>0}$ with

$$
J^{\psi, h}\left(\phi_{\epsilon}\right)<-8 \pi-8 \pi \log \pi-4 \pi \max _{y \in \Sigma}\left(2 \log h(y)+A_{y}\right)
$$

for sufficiently small $\epsilon$. This is a contradiction with (45), so no blowup happens, then we are in the position of Case a) and the proof terminates. In fact, our proof also shows that, if $J^{\psi, h}$ has no minimizer in $W^{1,2}(\Sigma, g)$, then

$$
\inf _{u \in W^{1,2}(\Sigma, g)} J^{\psi, h}(u)=-8 \pi-8 \pi \log \pi-4 \pi \max _{y \in \Sigma}\left(2 \log h(y)+A_{y}\right) .
$$


Proof of Theorem 3 —Part I: $h>0$. Suppose that $2 \log h(p)+A_{p}=\max _{y \in \Sigma}\left(2 \log h(y)+A_{y}\right)$. Let $r=\operatorname{dist}(x, p)$. Denote

$$
\begin{aligned}
\beta(r, \theta) & =G_{p}-\left(-4 \log r+A_{p}+b_{1} r \cos \theta+b_{2} r \sin \theta\right) \\
& =c_{1} r^{2} \cos ^{2} \theta+2 c_{2} r^{2} \cos \theta \sin \theta+c_{3} r^{2} \sin ^{2} \theta+O\left(r^{3}\right) .
\end{aligned}
$$

We define

$$
\phi_{\epsilon}= \begin{cases}-2 \log \left(r^{2}+\epsilon\right)+b_{1} r \cos \theta+b_{2} r \sin \theta+\log \epsilon, & r \leq \alpha \sqrt{\epsilon} \\ \left(G_{p}-\eta \beta(r, \theta)\right)+C_{\epsilon}+\log \epsilon, & \alpha \sqrt{\epsilon} \leq r \leq 2 \alpha \sqrt{\epsilon}, \\ G_{p}+C_{\epsilon}+\log \epsilon, & r \geq 2 \alpha \sqrt{\epsilon}\end{cases}
$$

where $\eta \in C_{0}^{1}\left(B_{2 \alpha \sqrt{\epsilon}}(p)\right)$ is a cutoff function satisfying $\eta \equiv 1$ in $B_{\alpha \sqrt{\epsilon}}(p)$ and $\left|\nabla_{g} \eta\right| \leq \frac{C}{\alpha \sqrt{\epsilon}}$, $C_{\epsilon}=-2 \log \frac{\alpha^{2}+1}{\alpha^{2}}-A_{p}, \alpha=\alpha(\epsilon)$ satisfying $\alpha \rightarrow \infty$ and $\alpha \sqrt{\epsilon} \rightarrow 0$ as $\epsilon \rightarrow 0$ will be determined later.

We denote by $(r, \theta)$ the chosen normal coordinate system around $p$. We write $g=d r^{2}+$ $g^{2}(r, \theta) d \theta^{2}$. It is well-known that

$$
g(r, \theta)=r-\frac{K_{g}(p)}{6} r^{3}+O\left(r^{4}\right) .
$$

Using (47) and calculating directly, we have

$$
\begin{aligned}
\int_{\Sigma}\left|\nabla_{g} \phi_{\epsilon}\right|^{2} d v_{g}= & 16 \pi \log \frac{\alpha^{2}+1}{\alpha^{2}}-16 \pi \log \epsilon-16 \pi+16 \pi \frac{1}{1+\alpha^{2}} \\
& +8 \pi A_{p}+\frac{16}{3} \pi K(p) \epsilon \log \left(\alpha^{2}+1\right)+O\left(\alpha^{4} \epsilon^{2} \log \left(\alpha^{2} \epsilon\right)\right)
\end{aligned}
$$

and

$$
\begin{aligned}
\int_{\Sigma} h e^{\phi_{\epsilon}} d v_{g}= & \pi h(p) \frac{\alpha^{2}}{\alpha^{2}+1}\left[1+\frac{1}{\alpha^{2}+1}-\frac{1}{6} K(p) \frac{\alpha^{2}+1}{\alpha^{2}} \epsilon \log \left(\alpha^{2}+1\right)\right. \\
& +\frac{1}{4} \frac{a^{2}+1}{\alpha^{2}}\left(b_{1}^{2}+b_{2}^{2}\right) \epsilon \log \left(\alpha^{2}+1\right)-\frac{1}{4} \frac{\alpha^{2}}{\alpha^{2}+1}\left(b_{1}^{2}+b_{2}^{2}\right) \epsilon \log \left(\alpha^{2} \epsilon\right) \\
& -\frac{1}{2} \frac{\alpha^{2}}{\alpha^{2}+1}\left(c_{1}+c_{3}-\frac{1}{3} K(p)\right) \epsilon \log \left(\alpha^{2} \epsilon\right) \\
& +\frac{1}{4} \frac{\alpha^{2}+1}{\alpha^{2}} \frac{\Delta_{g} h(p)}{h(p)} \epsilon \log \left(\alpha^{2}+1\right)-\frac{1}{4} \frac{\alpha^{2}}{\alpha^{2}+1} \frac{\Delta_{g} h(p)}{h(p)} \epsilon \log \left(\alpha^{2} \epsilon\right) \\
& \left.+\frac{1}{2} \frac{\alpha^{2}+1}{\alpha^{2}} \frac{k_{1} b_{1}+k_{2} b_{2}}{h(p)} \epsilon \log \left(\alpha^{2}+1\right)-\frac{1}{2} \frac{\alpha^{2}}{\alpha^{2}+1} \frac{k_{1} b_{1}+k_{2} b_{2}}{h(p)} \epsilon \log \left(\alpha^{2} \epsilon\right)\right] \\
& +O\left(\alpha^{4} \epsilon^{2}\right)+O(\epsilon) .
\end{aligned}
$$

We refer the readers to pages 241 and 245 in [9] for the details of calculation of (48) and (49).

Suppose that

$$
\psi(x)-\psi(p)=l_{1} r \cos \theta+l_{2} r \sin \theta+l_{3} r^{2} \cos ^{2} \theta+2 l_{4} r^{2} \sin \theta \cos \theta+l_{5} r^{2} \sin ^{2} \theta+O\left(r^{3}\right)
$$


in $B_{\delta}(p)$ for a small $\delta>0$.

Direct computations tell us that

$$
\int_{B_{\alpha \sqrt{\epsilon}}(p)}(\psi-\psi(p))\left(-2 \log \left(r^{2}+\epsilon\right)+b_{1} r \cos \theta+b_{2} r \sin \theta\right) d v_{g}=O\left(\alpha^{4} \epsilon^{2} \log \left(\alpha^{2} \epsilon\right)\right.
$$

and

$$
\begin{aligned}
& \int_{B_{\alpha \sqrt{\epsilon}}(p)}\left(-2 \log \left(r^{2}+\epsilon\right)+b_{1} r \cos \theta+b_{2} r \sin \theta\right) d v_{g} \\
= & -2 \pi \alpha^{2} \epsilon \log \left(\left(\alpha^{2}+1\right) \epsilon\right)-2 \pi \epsilon \log \left(\alpha^{2}+1\right)+2 \pi \alpha^{2} \epsilon+O\left(\alpha^{4} \epsilon^{2} \log \left(\alpha^{2} \epsilon\right) .\right.
\end{aligned}
$$

So

$$
\begin{aligned}
\int_{B_{\alpha \sqrt{\epsilon}}(p)} \psi \phi_{\epsilon} d v_{g}= & \psi(p)\left[-2 \pi \alpha^{2} \epsilon \log \left(\left(\alpha^{2}+1\right) \epsilon\right)-2 \pi \epsilon \log \left(\alpha^{2}+1\right)+2 \pi \alpha^{2} \epsilon\right] \\
& +\log \epsilon \int_{B_{\alpha \sqrt{\epsilon}}(p)} \psi d v_{g}+O\left(\alpha^{4} \epsilon^{2} \log \left(\alpha^{2} \epsilon\right) .\right.
\end{aligned}
$$

We have

$$
\begin{aligned}
& \int_{\Sigma \backslash B_{\alpha \sqrt{\epsilon}}(p)} \psi\left(\left(G_{p}-\eta \beta(r, \theta)\right)+C_{\epsilon}+\log \epsilon\right) d v_{g} \\
= & \int_{\Sigma \backslash B_{\alpha \sqrt{\epsilon}}(p)} \psi G_{p} d v_{g}-\int_{B_{2 \alpha \sqrt{\epsilon}}(p) \backslash B_{\alpha \sqrt{\epsilon}}(p)} \psi \eta \beta(r, \theta) d v_{g}+\left(C_{\epsilon}+\log \epsilon\right) \int_{\Sigma \backslash B_{\alpha \sqrt{\epsilon}}(p)} \psi d v_{g} .
\end{aligned}
$$

By a direct calculation, one has

$$
\begin{aligned}
\int_{\Sigma \backslash B_{\alpha \sqrt{\epsilon}}(p)} \psi G_{p} d v_{g} & =-\int_{B_{\alpha \sqrt{\epsilon}}(p)} \psi G_{p} d v_{g} \\
& =-\int_{B_{\alpha \sqrt{\epsilon}}(p)}(\psi-\psi(p)) G_{p} d v_{g}-\int_{B_{\alpha \sqrt{\epsilon}}(p)} \psi(p) G_{p} d v_{g} \\
& =-\psi(p)\left(-2 \pi \alpha^{2} \epsilon \log \left(\alpha^{2} \epsilon\right)+2 \pi \alpha^{2} \epsilon+\pi A_{p} \alpha^{2} \epsilon\right)+O\left(\alpha^{4} \epsilon^{2} \log \left(\alpha^{2} \epsilon\right)\right.
\end{aligned}
$$

where we have used

$$
\int_{B_{\alpha \sqrt{\epsilon}}(p)} \psi d v_{g}=\pi \psi(p) \alpha^{2} \epsilon+O\left(\alpha^{4} \epsilon^{2}\right)
$$

Meanwhile we have

$$
-\int_{B_{2 \alpha \sqrt{\epsilon}}(p) \backslash B_{\alpha \sqrt{\epsilon}}(p)} \psi \eta \beta(r, \theta) d v_{g}=O\left(\alpha^{4} \epsilon^{2}\right) .
$$

Inserting (52) and (53) into (51), we have

$$
\begin{aligned}
\int_{\Sigma \backslash B_{\alpha \sqrt{\epsilon}}(p)} \psi \phi_{\epsilon} d v_{g}= & -\psi(p)\left(-2 \pi \alpha^{2} \epsilon \log \left(\alpha^{2} \epsilon\right)+2 \pi \alpha^{2} \epsilon+\pi A_{p} \alpha^{2} \epsilon\right) \\
& +\left(C_{\epsilon}+\log \epsilon\right) \int_{\Sigma \backslash B_{\alpha \sqrt{\epsilon}}(p)} \psi d v_{g}+O\left(\alpha^{4} \epsilon^{2} \log \left(\alpha^{2} \epsilon\right) .\right.
\end{aligned}
$$


Combining (50) and (54), we have

$$
\begin{aligned}
\int_{\Sigma} \psi \phi_{\epsilon} d v_{g}= & \int_{B_{\alpha \sqrt{\epsilon}}(p)} \psi \phi_{\epsilon} d v_{g}+\int_{\Sigma \backslash B_{\alpha \sqrt{\epsilon}}(p)} \psi \phi_{\epsilon} d v_{g} \\
= & \int_{\Sigma} \psi d v_{g}\left(\log \epsilon-2 \log \frac{\alpha^{2}+1}{\alpha^{2}}-A(p)-2 \pi \frac{\psi(p)}{\int_{\Sigma} \psi d v_{g}} \epsilon \log \left(\alpha^{2}+1\right)\right) \\
& +O\left(\alpha^{4} \epsilon^{2} \log \left(\alpha^{2} \epsilon\right) .\right.
\end{aligned}
$$

Then by 48, 49, and (55) one has

$$
\begin{aligned}
J^{\psi, h}\left(\phi_{\epsilon}\right)= & \frac{1}{2} \int_{\Sigma}\left|\nabla_{g} \phi_{\epsilon}\right|^{2} d v_{g}+8 \pi \frac{1}{\int_{\Sigma} \psi d v_{g}} \int_{\Sigma} \psi \phi_{\epsilon} d v_{g}-8 \pi \log \int_{\Sigma} h e^{\phi_{\epsilon}} d v_{g} \\
= & -8 \pi-8 \pi \log \pi-4 \pi A_{p}-8 \pi \log h(p) \\
& -16 \pi^{2}\left(\frac{\psi(p)}{\int_{\Sigma} \psi d v_{g}}-\frac{1}{4 \pi} K_{g}(p)\right) \epsilon \log \left(\alpha^{2}+1\right)+16 \pi^{2}\left(1-\frac{1}{4 \pi} K_{g}(p)\right) \epsilon \log \left(\alpha^{2} \epsilon\right) \\
& -2 \pi \frac{\alpha^{2}+1}{\alpha^{2}}\left(b_{1}^{2}+b_{2}^{2}\right) \epsilon \log \left(\alpha^{2}+1\right)+2 \pi \frac{\alpha^{2}}{\alpha^{2}+1}\left(b_{1}^{2}+b_{2}^{2}\right) \epsilon \log \left(\alpha^{2} \epsilon\right) \\
& -2 \pi \frac{\alpha^{2}+1}{\alpha^{2}} \frac{\Delta_{g} h(p)}{h(p)} \epsilon \log \left(\alpha^{2}+1\right)+2 \pi \frac{\alpha^{2}}{\alpha^{2}+1} \frac{\Delta_{g} h(p)}{h(p)} \epsilon \log \left(\alpha^{2} \epsilon\right) \\
& -4 \pi \frac{\alpha^{2}+1}{\alpha^{2}} \frac{k_{1} b_{1}+k_{2} b_{2}}{h(p)} \epsilon \log \left(\alpha^{2}+1\right)+4 \pi \frac{\alpha^{2}}{\alpha^{2}+1} \frac{k_{1} b_{1}+k_{2} b_{2}}{h(p)} \epsilon \log \left(\alpha^{2} \epsilon\right) \\
& +O\left(\frac{\epsilon \log \left(\alpha^{2}+1\right)}{\alpha^{2}}\right)+O\left(\frac{-\epsilon \log \left(\alpha^{2} \epsilon\right)}{\alpha^{2}}\right)+O\left(\left(\epsilon \log \left(\alpha^{2}+1\right)\right)^{2}\right) \\
& +O\left(\left(-\epsilon \log \left(\alpha^{2} \epsilon\right)\right)^{2}\right)+O\left(\frac{1}{\alpha^{4}}\right)+O\left(\alpha^{4} \epsilon^{2}\right)+O(\epsilon),
\end{aligned}
$$

where we have used Proposition 3.2 in [9], i.e., $c_{1}+c_{3}+\frac{2}{3} K_{g}(p)=4 \pi$. By choosing $\alpha=$ $(\epsilon \log (-\log \epsilon))^{-1 / 4}$, we have

$$
\begin{aligned}
J^{\psi, h}\left(\phi_{\epsilon}\right)= & -8 \pi-8 \pi \log \pi-4 \pi A_{p}-8 \pi \log h(p) \\
& -16 \pi^{2}\left(\frac{1}{2}\left(\frac{\psi(p)}{\int_{\Sigma} \psi d v_{g}}+1\right)-\frac{1}{4 \pi} K_{g}(p)+\frac{b_{1}^{2}+b_{2}^{2}}{8 \pi}+\frac{\Delta_{g} h(p)}{8 \pi h(p)}+\frac{k_{1} b_{1}+k_{2} b_{2}}{4 \pi h(p)}\right) \epsilon(-\log \epsilon) \\
& +o(\epsilon(-\log \epsilon)) .
\end{aligned}
$$

So if (8) is satisfied, by (56) we have

$$
J^{\psi, h}\left(\phi_{\epsilon}\right)<-8 \pi-8 \pi \log \pi-4 \pi \max _{y \in \Sigma}\left(2 \log h(y)+A_{y}\right)
$$

for sufficiently small $\epsilon>0$. This ends the proof of Theorem 3 when $h>0$.

\section{Proof of Theorem 3—Part II: $h \geq 0, h \neq 0$}

In this section, we shall deal with the situation that $h \geq 0$ and $h \not \equiv 0$ on $\Sigma$ and end the proof of Theorem 3 The idea comes from our paper [25]. 
First, we need the following concentration lemma, which can be seen as a generalization of one on $S^{2}$ proved by Chang-Yang [5] and one on a general compact Riemannian surface proved by Ding-Jost-Li-Wang [10].

Proposition 14. Let $(\Sigma, g)$ be a compact Riemannian surface. Let $\psi$ be a smooth function on $\Sigma$ satisfying $\int_{\Sigma} \psi d v_{g} \neq 0$. Given a sequence of $u_{j} \in W^{1,2}(\Sigma, g)$ with $\int_{\Sigma} e^{u_{j}} d v_{g}=1$ and

$$
\frac{1}{2} \int_{\Sigma}\left|\nabla_{g} u_{j}\right|^{2} d v_{g}+8 \pi \frac{1}{\int_{\Sigma} \psi d v_{g}} \int_{\Sigma} \psi u_{j} d v_{g} \leq C
$$

Then either

(i) there exists a constant $C_{0}>0$ such that $\int_{\Sigma}\left|\nabla_{g} u_{j}\right|^{2} d v_{g} \leq C_{0}$ or

(ii) there exists a subsequence which is also denoted by $u_{j}$ concentrates at a point $p \in \Sigma$, i.e., for any $r>0$,

$$
\lim _{j \rightarrow \infty} \int_{B_{r}(p)} e^{u_{j}} d v_{g}=1
$$

To prove Proposition 14, one needs the following "distribution of mass" lemma, which can be seen as a generalization of one proved by Aubin [2] (see also [7]).

Lemma 15. Let $(\Sigma, g)$ be a compact Riemannian surface. Let $\psi$ be a smooth function on $\Sigma$ satisfying $\int_{\Sigma} \psi d v_{g} \neq 0$. Let $\Omega_{1}$ and $\Omega_{2}$ be two subsets of $\Sigma$ satisfying $\operatorname{dist}\left(\Omega_{1}, \Omega_{2}\right) \geq \epsilon_{0}>0$ and $\alpha_{0} \in(0,1 / 2)$. For any $\epsilon \in(0,1)$, there exists a constant $C=C\left(\epsilon, \epsilon_{0}, \alpha_{0}\right)$ such that

$$
\int_{\Sigma} e^{u} d v_{g} \leq C \exp \left\{\frac{1}{32 \pi(1-\epsilon)}\left\|\nabla_{g} u\right\|_{2}^{2}+\widetilde{u}\right\}
$$

holds for any $u \in W^{1,2}(\Sigma, g)$ satisfying

$$
\frac{\int_{\Omega_{1}} e^{u} d v_{g}}{\int_{\Sigma} e^{u} d v_{g}} \geq \alpha_{0} \quad \text { and } \quad \frac{\int_{\Omega_{2}} e^{u} d v_{g}}{\int_{\Sigma} e^{u} d v_{g}} \geq \alpha_{0} .
$$

Proof. Let $\phi_{1}, \phi_{2}$ be two smooth functions on $\Sigma$ such that

$$
0 \leq \phi_{i} \leq 1, \phi_{i} \equiv 1, \text { for } x \in \Omega_{i}, i=1,2
$$

and supp $\phi_{1} \cap \operatorname{supp} \phi_{2}=\emptyset$. It suffices to show that for $u \in W^{1,2}(\Sigma, g), \widetilde{u}=0$, (57) implies

$$
\int_{\Sigma} e^{u} d v_{g} \leq C \exp \left\{\frac{1}{32 \pi(1-\epsilon)}\left\|\nabla_{g} u\right\|_{2}^{2}\right\} .
$$

We can assume without loss of generality that $\left\|\nabla_{g}\left(\phi_{1} u\right)\right\|_{2} \leq\left\|\nabla_{g}\left(\phi_{2} u\right)\right\|_{2}$. Then by (57) and Theorem 1, one has

$$
\begin{aligned}
\int_{\Sigma} e^{u} d v_{g} & \leq \frac{1}{\alpha_{0}} \int_{\Omega_{1}} e^{u} d v_{g} \leq \frac{1}{\alpha_{0}} \int_{\Sigma} e^{\phi_{1} u} d v_{g} \\
& \leq \frac{C}{\alpha_{0}} \exp \left\{\frac{1}{16 \pi}\left\|\nabla_{g}\left(\phi_{1} u\right)\right\|_{2}^{2}+\widetilde{\phi_{1} u}\right\} \\
& \leq \frac{C}{\alpha_{0}} \exp \left\{\frac{1}{32 \pi}\left\|\nabla_{g}\left[\left(\phi_{1}+\phi_{2}\right) u\right]\right\|_{2}^{2}+\widetilde{\phi_{1} u}\right\} \\
& \leq \frac{C\left(\epsilon_{0}\right)}{\alpha_{0}} \exp \left\{\frac{1}{32 \pi}\left(1+\epsilon_{1}\right)\left\|\nabla_{g} u\right\|_{2}^{2}+C\left(\epsilon_{1}\right)\|u\|_{2}^{2}\right\}
\end{aligned}
$$


for some small $\epsilon_{1}>0$, where in the last inequality we have used Lemma 5 and the Cauchy's inequality.

Using the condition $\widetilde{u}=0$ one can get rid of the term $\|u\|_{2}^{2}$ on the right hand side of (59).

Given small enough $\eta>0$, there exists $a_{\eta}$ such that $\operatorname{Vol}_{g}\left\{x \in \Sigma: u(x) \geq a_{\eta}\right\}=\eta$. Applying (59) to the function $\left(u-a_{\eta}\right)_{+}=\max \left\{0,\left(u-a_{\eta}\right)\right\}$, we have

$$
\begin{aligned}
\int_{\Sigma} e^{u} d v_{g} & \leq e^{a_{\eta}} \int_{\Sigma} e^{\left(u-a_{\eta}\right)} d v_{g} \leq e^{a_{\eta}} \int_{\Sigma} e^{\left(u-a_{\eta}\right)_{+}} d v_{g} \\
& \leq C \exp \left\{\frac{1}{32 \pi}\left(1+\epsilon_{1}\right)\left\|\nabla_{g} u\right\|_{2}^{2}+C\left(\epsilon_{1}\right)\left\|\left(u-a_{\eta}\right)_{+}\right\|_{2}^{2}+a_{\eta}\right\},
\end{aligned}
$$

where $C=C\left(\epsilon_{0}, \alpha_{0}\right)$.

By the Hölder inequality and Lemma 6 , we have

$$
\begin{aligned}
\int_{\Sigma}\left|\left(u-a_{\eta}\right)_{+}\right|^{2} d v_{g} & =\int_{\left\{x \in \Sigma: u(x) \geq a_{\eta}\right\}}\left|\left(u-a_{\eta}\right)_{+}\right|^{2} d v_{g} \\
& \leq\left(\int_{\left\{x \in \Sigma: u(x) \geq a_{\eta}\right\}}\left|\left(u-a_{\eta}\right)_{+}\right|^{4}\right)^{1 / 2} \cdot \eta^{1 / 2} \\
& \leq\left(\int_{\Sigma}|u|^{4} d v_{g}\right)^{1 / 2} \cdot \eta^{1 / 2} \\
& \leq C \int_{\Sigma}\left|\nabla_{g} u\right|^{2} d v_{g} \cdot \eta^{1 / 2} .
\end{aligned}
$$

By the Höder inequality and Lemma 5 , we have

$$
a_{\eta} \cdot \eta \leq \int_{\left\{x \in \Sigma: u(x) \geq a_{\eta}\right\}} u d v_{g} \leq \int_{\Sigma}|u| d v_{g} \leq C\left(\int_{\Sigma}\left|\nabla_{g} u\right|^{2} d v_{g}\right)^{1 / 2} .
$$

So

$$
a_{\eta} \leq \eta \int_{\Sigma}\left|\nabla_{g} u\right|^{2} d v_{g}+\frac{C}{\eta} .
$$

Substituting (61) and (62) into (60) and choosing $\epsilon_{1}$ and $\eta$ sufficiently small such that

$$
\frac{1}{32 \pi}\left(1+\epsilon_{1}\right)+C\left(\epsilon_{1}\right) C \eta^{1 / 2}+\eta \leq \frac{1}{32 \pi(1-\epsilon)},
$$

then we obtain the inequaliaty (58). This ends the proof of Lemma 15 .

Proof of Proposition 14 If (ii) does not hold, i.e., every subsequence of $u_{j}$ does not concentrate. Then for any $p \in \Sigma$ there exists $r \in\left(0, i_{\Sigma} / 16\right)$ such that

$$
\lim _{j \rightarrow \infty} \int_{B_{r}(p)} e^{u_{j}} d v_{g}<\delta_{0}<1,
$$

where $i_{\Sigma}$ is the injective radius of $(\Sigma, g)$. (Note that we do not distinguish sequence and its subsequences.) 
Since $(\Sigma, g)$ is compact, there exists a finite set $\left\{\left(p_{l}, r_{l}\right): l=1,2, \ldots, L\right\}$ satisfying

$$
\lim _{j \rightarrow \infty} \int_{B_{r_{l}}\left(p_{l}\right)} e^{u_{j}} d v_{g}<\delta<1
$$

and $\bigcup_{l=1}^{L} B_{r_{l}}\left(p_{l}\right)=\Sigma$.

Without loss of generality, one may assume that

$$
\lim _{j \rightarrow \infty} \int_{B_{r_{1}}\left(p_{1}\right)} e^{u_{j}} d v_{g} \geq \alpha_{0}>0
$$

where $\alpha_{0} \in\left(0, \delta_{0}\right)$ is a constant.

We prove $(i)$ must happen by contradiction. Suppose (i) not happens, i.e., $\int_{\Sigma}\left|\nabla_{g} u_{j}\right|^{2} d v_{g}$ is unbounded. Then from Lemma 15 we know

$$
\lim _{j \rightarrow \infty} \int_{\Sigma \backslash B_{2 r_{1}}\left(p_{1}\right)} e^{u_{j}} d v_{g}=0 .
$$

Choosing a normal coordinate system $\left(x_{1}, x_{2}\right)$ around $p_{1}$ and assuming in $B_{16 r_{1}}\left(p_{1}\right)$

$$
\frac{1}{2}|x-y| \leq \operatorname{dist}_{g}(x, y) \leq 2|x-y|
$$

where $|x-y|=\operatorname{dist}_{\mathbb{R}^{2}}(x, y)$.

We consider the square $P_{1}=\left\{\left|x_{i}\right| \leq 4 r_{1}: i=1,2\right\} \subset \mathbb{R}^{2}$, from 64 one knows

$$
\lim _{j \rightarrow \infty} \int_{\exp _{p_{1}}\left(P_{1}\right)} e^{u_{j}} d v_{g}=1 .
$$

Dividing $P_{1}$ into 16 equal sub-squares. Since $\int_{\Sigma}\left|\nabla_{g} u_{j}\right|^{2} d v_{g}$ is unbounded, by Lemma[15] one gets a square $P_{2}$ which is a union of at most 9 of the equal sub-squares of $P_{1}$ such that

$$
\lim _{j \rightarrow \infty} \int_{\exp _{p_{1}}\left(P_{2}\right)} e^{u_{j}} d v_{g}=1 .
$$

Continuing this procedure, we can obtain a sequence of square $P_{n}$. It is easy to check that $P_{n} \rightarrow p_{0}$ as $n \rightarrow \infty$ for some $p_{0} \in \Sigma$ and

$$
\lim _{j \rightarrow \infty} \int_{B_{r}\left(p_{0}\right)} e^{u_{j}} d v_{g}=1
$$

for any $r \in\left(0, i_{\Sigma}\right)$. This contradicts $(63)$. The contradiction tells us that $\int_{\Sigma}\left|\nabla_{g} u_{j}\right|^{2} d v_{g}$ is bounded, i.e., (i) holds. This ends the proof of Proposition 14.

Now, we are ready to prove Theorem 3 when $h \geq 0, h \not \equiv 0$.

Proof of Theorem 3-Part II: $h \geq 0, h \neq 0$. Checking the proof of Theorem 3-Part I: $h>0$ carefully, one will finds that the condition $h>0$ is just used in solving the bubble (21). Therefore, if $h \geq 0, h \neq 0$, we just need to prove that the blowup (if happens) will not happen on zero point of $h$. 
In the following we assume $u_{\epsilon}$ blows up, i.e., $\left\|\nabla u_{\epsilon}\right\|_{2} \rightarrow+\infty$ as $\epsilon \rightarrow 0$.

Recalling that in (5), we have $u_{\epsilon} \in C^{\infty}(\Sigma) \cap \widetilde{X}$. Lemma 9 still holds, i.e., $c_{\epsilon} \rightarrow \infty$ as $\epsilon \rightarrow 0$. Let $\Omega \subset \Sigma$ be a domain. If $\lambda_{\epsilon}^{-1} \int_{\Omega} h e^{u_{\epsilon}} d v_{g} \leqslant \frac{1}{2}-\delta$ for some $\delta \in\left(0, \frac{1}{2}\right)$, then (28) implies that

$$
\left\|u_{\epsilon}\right\|_{L^{\infty}\left(\Omega_{0}\right)} \leq C\left(\Omega_{0}, \Omega\right), \quad \forall \Omega_{0} \subset \subset \Omega .
$$

Suppose $x_{\epsilon} \rightarrow p$ as $\epsilon \rightarrow 0$. As we explained before, to prove Theorem 3 it suffices to prove that $h(p)>0$. For this purpose, we set $v_{\epsilon}=u_{\epsilon}-\log \int_{\Sigma} e^{u_{\epsilon}} d v_{g}$. Then we have

$$
\int_{\Sigma} e^{v_{\epsilon}} d v_{g}=1, \quad J_{\epsilon}^{\psi, h}\left(v_{\epsilon}\right)=J_{\epsilon}^{\psi, h}\left(u_{\epsilon}\right)
$$

and

$$
J_{\epsilon}^{\psi, h}\left(u_{\epsilon}\right)=\inf _{u \in \widetilde{X}} J_{\epsilon}^{\psi, h}(u) \leq J_{\epsilon}^{\psi, h}(0)=-8 \pi(1-\epsilon) \log \int_{\Sigma} h d v_{g} .
$$

The Hölder inequality together with Lemmas 5 and 7 tells us that

$$
\left|\bar{u}_{\epsilon}\right|=\left|\widetilde{u}_{\epsilon}-\bar{u}_{\epsilon}\right|=\left|\frac{1}{\int_{\Sigma} \psi d v_{g}} \int_{\Sigma} \psi\left(u_{\epsilon}-\bar{u}_{\epsilon}\right) d v_{g}\right| \leq C
$$

By Jensen's inequality and (68), we have

$$
\begin{aligned}
\widetilde{v}_{\epsilon}=-\log \int_{\Sigma} e^{u_{\epsilon}} d v_{g} & =-\log \operatorname{Vol}_{g}(\Sigma)-\log \left(\frac{1}{\operatorname{Vol}_{g}(\Sigma)} \int_{\Sigma} e^{u_{\epsilon}} d v_{g}\right) \\
& \leq-\log \operatorname{Vol}_{g}(\Sigma)+C \\
& \leq C .
\end{aligned}
$$

Combining (66), 67) and (69) one obtains

$$
\begin{aligned}
\frac{1}{2} \int_{\Sigma}\left|\nabla_{g} v_{\epsilon}\right|^{2} d v_{g}+8 \pi \widetilde{v}_{\epsilon} & \leq J_{\epsilon}^{\psi, h}\left(v_{\epsilon}\right)+8 \pi \epsilon \widetilde{v}_{\epsilon}+8 \pi(1-\epsilon) \log \int_{\Sigma} h e^{v_{\epsilon}} d v_{g} \\
& \leq J_{\epsilon}^{\psi, h}\left(u_{\epsilon}\right)+8 \pi \epsilon \widetilde{v}_{\epsilon}+8 \pi(1-\epsilon) \log \max _{\Sigma} h \\
& \leq C+8 \pi(1-\epsilon) \log \frac{\max _{\Sigma} h}{\int_{\Sigma} h d v_{g}}
\end{aligned}
$$

$\leq C$.

Clearly, (ii) of Lemma 14 holds in this case. Hence there exists some $p^{\prime} \in \Sigma$ such that $v_{\epsilon}$ concentrates at $p^{\prime}$, namely,

$$
\lim _{\epsilon \rightarrow 0} \int_{B_{r}\left(p^{\prime}\right)} e^{v_{\epsilon}} d v_{g}=1, \quad \forall r>0 .
$$

We first claim that

$$
h\left(p^{\prime}\right)>0 .
$$


To see this, in view of (70), we calculate

$$
\begin{aligned}
\frac{\int_{\Sigma} h e^{u_{\epsilon}} d v_{g}}{\int_{\Sigma} e^{u_{\epsilon}} d v_{g}}=\int_{\Sigma} h e^{v_{\epsilon}} d v_{g} & =\int_{B_{r}\left(p^{\prime}\right)} h e^{v_{\epsilon}} d v_{g}+\int_{\Sigma \backslash B_{r}\left(p^{\prime}\right)} h e^{v_{\epsilon}} d v_{g} \\
& =\left(h\left(p^{\prime}\right)+o_{r}(1)\right) \int_{B_{r}\left(p^{\prime}\right)} e^{v_{\epsilon}} d v_{g}+o_{\epsilon}(1) \\
& =\left(h\left(p^{\prime}\right)+o_{r}(1)\right)\left(1+o_{\epsilon}(1)\right)+o_{\epsilon}(1) \\
& =h\left(p^{\prime}\right)+o_{r}(1)+o_{\epsilon}(1) .
\end{aligned}
$$

Because the left hand side of (72) does not depend on $r$, we have by passing to the limit $r \rightarrow 0$,

$$
\frac{\int_{\Sigma} h e^{u_{\epsilon}} d v_{g}}{\int_{\Sigma} e^{u_{\epsilon}} d v_{g}}=h\left(p^{\prime}\right)+o_{\epsilon}(1) .
$$

So by (73) and Theorem 1 we have

$$
\begin{aligned}
J_{\epsilon}^{\psi, h}\left(u_{\epsilon}\right)= & \frac{1}{2} \int_{\Sigma}\left|\nabla_{g} u_{\epsilon}\right|^{2} d v_{g}-8 \pi(1-\epsilon) \log \int_{\Sigma} h e^{u_{\epsilon}} d v_{g} \\
= & \frac{1}{2} \int_{\Sigma}\left|\nabla_{g} u_{\epsilon}\right|^{2} d v_{g}-8 \pi(1-\epsilon) \log \left(h\left(p^{\prime}\right)+o_{\epsilon}(1)\right) \\
& -8 \pi(1-\epsilon) \log \int_{\Sigma} e^{u_{\epsilon}} d v_{g} \\
\geq & \frac{1}{2} \int_{\Sigma}\left|\nabla_{g} u_{\epsilon}\right|^{2} d v_{g}-8 \pi(1-\epsilon) \log \left(h\left(p^{\prime}\right)+o_{\epsilon}(1)\right) \\
& -8 \pi(1-\epsilon) \log \left(C \exp \left\{\frac{1}{16 \pi} \int_{\Sigma}\left|\nabla_{g} u_{\epsilon}\right|^{2} d v_{g}\right\}\right) \\
\geq & -8 \pi(1-\epsilon) \log \left(C\left(h\left(p^{\prime}\right)+o_{\epsilon}(1)\right)\right) .
\end{aligned}
$$

Combining (67) and (74), we obtain

$$
-8 \pi(1-\epsilon) \log \int_{\Sigma} h d v_{g} \geq-8 \pi(1-\epsilon) \log \left(C\left(h\left(p^{\prime}\right)+o_{\epsilon}(1)\right)\right),
$$

and whence

$$
\log \int_{\Sigma} h d v_{g} \leq \log \left(C h\left(p^{\prime}\right)\right)
$$

This immediately leads to (71).

Then we claim that

$$
p^{\prime}=p
$$

In view of (ii) of Lemma 14, there holds $\int_{\Omega} e^{v_{\epsilon}} d v_{g} \rightarrow 0, \forall \Omega \subset \subset \Sigma \backslash\left\{p^{\prime}\right\}$. Noting that $u_{\epsilon} \in \widetilde{X}$, from (73) we have

$$
\lambda_{\epsilon}^{-1} \int_{\Omega} h e^{u_{\epsilon}} d v_{g}=\lambda_{\epsilon}^{-1} \int_{\Sigma} e^{u_{\epsilon}} d v_{g} \int_{\Omega} h e^{v_{\epsilon}} d v_{g} \leq \frac{\max _{\Sigma} h}{h\left(p^{\prime}\right)+o_{\epsilon}(1)} \int_{\Omega} e^{v_{\epsilon}} d v_{g} \rightarrow 0
$$

as $\epsilon \rightarrow 0$. Combining (65) and (76), we obtain $\left\|u_{\epsilon}\right\|_{L^{\infty}(\Omega)} \leq C$ and thus $\left\|v_{\epsilon}-\widetilde{v}_{\epsilon}\right\|_{L^{\infty}(\Omega)} \leq C$ for any $\Omega \subset \subset \backslash\left\{p^{\prime}\right\}$. This together with (69) implies that $v_{\epsilon}(x) \leq C$ for all $x \in \Omega \subset \subset \backslash\left\{p^{\prime}\right\}$. 
It follows from (73) and $u_{\epsilon} \in \widetilde{X}$ that

$$
\lambda_{\epsilon}^{-1} \int_{\Sigma} e^{u_{\epsilon}} d v_{g}=\frac{1}{h\left(p^{\prime}\right)+o_{\epsilon}(1)}<\frac{2}{h\left(p^{\prime}\right)}
$$

for sufficiently small $\epsilon>0$. Suppose $p^{\prime} \neq p$. Recalling that $c_{\epsilon}=u_{\epsilon}\left(x_{\epsilon}\right)=\max _{\Sigma} u_{\epsilon}$ and $x_{\epsilon} \rightarrow p$, we find a domain $\Omega$ such that $x_{\epsilon} \in \Omega \subset \subset \Sigma \backslash\left\{p^{\prime}\right\}$. Hence

$$
c_{\epsilon}-\log \lambda_{\epsilon}=u_{\epsilon}\left(x_{\epsilon}\right)-\log \lambda_{\epsilon}=v_{\epsilon}\left(x_{\epsilon}\right)+\log \left(\lambda_{\epsilon}^{-1} \int_{\Sigma} e^{u_{\epsilon}} d v_{g}\right) \leq C,
$$

which contradicts Lemma 9 and concludes our claim (75). Combining (71) and (75), we obtain $h(p)>0$. The remaining part of the proof of Theorem 3 is completely analogous to Section 4 , we omit the details here.

Acknowledgements. The work is supported by the National Science Foundation of China (Grant No. 11401575). The author thanks Professor Yunyan Yang for his helpful discussions and suggestions.

\section{References}

[1] Adimurthi, O. Druet, Blow-up analysis in dimension 2 and a sharp form of Trudinger-Moser inequality, Comm. Partial Differential Equations 29 (2004) 295-322.

[2] T. Aubin, Nonlinear analysis on manifolds, Spinger-Verlag, New York, 1982.

[3] D. Bartolucci, C. Lin, Existence and uniqueness for mean field equations on multiply connected domains at the critical parameter, Math. Ann. 359 (2014), 1-44.

[4] H. Brezis, F. Merle, Uniform estimates and blow-up behavior for solutions of $-\Delta u=V(x) e^{u}$ in two dimensions, Comm. Partial Differential Equations 16 (1991) 1223-1253.

[5] S.-Y. A. Chang, P. Yang, Conformal deformation of metrics on $S^{2}$, J. Diff. Geom. 23 (1988) 259-296.

[6] W. Chen, C. Li, Classification of solutions of some nonlinear elliptic equations, Duke Math. J. 63 (1991) 615-622.

[7] W. Chen, C. Li, Prescribing Gaussian curvatures on surfaces with conical singularities, J. Geom. Anal. 1 (1991) 359-372.

[8] X. Chen, M. Zhu, Liouville energy on a topological two sphere, Commun. Math. Stat. 1 (2013), no. 4, 369-385.

[9] W. Ding, J. Jost, J. Li, G. Wang, The differential equation $\Delta u=8 \pi-8 \pi h e^{u}$ on a compact Riemann surface, Asian J. Math. 1 (1997) 230-248.

[10] W. Ding, J. Jost, J. Li, G. Wang, An analysis of the two-vortex case in the Chern-Simons Higgs model, Calc. Var. 7 (1998) 87-97.

[11] L. Fontana, Sharp borderline Sobolev inequalities on compact Riemannian manifolds, Comment. Math. Helv. 68 (1993) 415-454.

[12] D. Gilbarg, N. S. Trudinger, Elliptic partial differential equations of second order, Springer-Verlag, Berlin, 2001.

[13] E. Hebey, Nonlinear analysis on manifolds: Sobolev spaces and inequalities, Courant Lecture Notes in Mathematics, 5 .

[14] J. Kazdan, F. Warner, Curvature functions for compact 2-manifolds, Ann. of Math. 99 (1974) 14-47.

[15] C. Lin, C. Wang, Elliptic functions, Green functions and the mean field equations on tori. Ann. Math. 172(2) (2010) 911-954.

[16] G. Lu, Y. Yang, The sharp constant and extremal functions for Trudinger-Moser inequalities involving $L^{p}$ norms, Discrete and Continuous Dynamical Systems 25 (2009) 963-979.

[17] C. Tintarev, Trudinger-Moser inequality with remainder terms, J. Funct. Anal. 266 (2014) 55-66.

[18] G. Wang, D. Ye, A Hardy-Moser-Trudinger inequality, Adv. Math. 230 (2012), no. 1, 294-320.

[19] G. Wang, C. Xia, Blow-up analysis of a Finsler-Liouville equation in two dimensions, J. Differential Equations 252 (2012), no. 2, 1668-1700.

[20] M. Wang, Q. Liu, The equation $\Delta u+\nabla \phi \cdot \nabla u=8 \pi c\left(1-h e^{u}\right)$ on a Riemann surface, J. Partial Differential Equations 25 (2001), no. 4, 335-355.

[21] Y. Yang, A sharp form of the Moser-Trudinger inequality on a compact Riemannian surface, Trans. Amer. Math. Soc. 359 (2007) 5761-5776. 
[22] Y. Yang, Extremal functions for Trudinger-Moser inequalities of Adimurthi-Druet type in dimension two, J. Differential Equations 258 (2015) 3161-3193.

[23] Y. Yang, A Trudinger-Moser inequality on compact Riemannian surface involving Gaussian curvature, J. Geom. Anal. 26 (2016), no. 4, 2893-2913.

[24] Y. Yang, X. Zhu, An improved Hardy-Trudinger-Moser inequality, Ann. Global Anal. Geom. 49 (2016), no. 1, 23-41.

[25] Y. Yang, X. Zhu, A remark on a result of Ding-Jost-Li-Wang, arXiv:1610.00774 to be published on Proceedings of the American Mathematical Society.

[26] X. Zhu, A weak Trudinger-Moser inequality with a singular weight on compact Riemannian surface, submitted. 